\title{
CORRECTIONS
}

\section{Correcting refractive error in low income countries}

In this editorial by Lisa Keay and David S Friedman (BMJ 2011;343:d4793, doi:10.1136/bmj.d4793) the authors mixed up their workplace affiliations. The workplace affiliation for
Lisa Keay should have been the one given for David S Friedman, and vice versa.

Cite this as: $B M J 2011 ; 343: d 5195$ 